\title{
Spontaneous regression of a vein of Galen aneurysmal malformation in a pediatric patient: illustrative case
}

\author{
Kevin K. Kumar, MD, PhD, ${ }^{1}$ Linden E. Fornoff, MD, ${ }^{3}$ Robert L. Dodd, MD, PhD, ${ }^{1}$ Michael P. Marks, MD, ${ }^{2}$ and David S. Hong, MD \\ Departments of ${ }^{1}$ Neurosurgery and ${ }^{2}$ Radiology, Stanford University, Stanford, California; and ${ }^{3}$ Boys Town National Research Hospital, Boys Town, Nebraska
}

\begin{abstract}
BACKGROUND Vein of Galen aneurysmal malformations (VGAMs) are rare congenital intracranial vascular lesions that represent $30 \%$ of all pediatric vascular anomalies. These lesions are associated with severe manifestations, including congestive heart failure, hydrocephalus, and spontaneous hemorrhage. The mainstay of management is medical stabilization followed by endovascular embolization of the lesion. Although VGAM was first reported in 1937, there are few published cases demonstrating spontaneous regression of the lesion.

OBSERVATIONS The authors report the case of a 31-month-old female who presented with an incidentally found VGAM. After initial evaluation, including magnetic resonance imaging and angiography, the patient was lost to follow-up. Upon her return to the clinic at age 12 years, the previously identified VGAM was absent, indicative of involution of the lesion. The patient remained asymptomatic and met appropriate developmental milestones during this interval.

LESSONS This report adds a rare case of the spontaneous resolution of VGAM to the literature. This case may suggest the presence of VGAMs that are asymptomatic, undetected, and regress within the pediatric population. Future studies may benefit from identifying imaging and angiographic findings predictive of spontaneous regression. There may be a role for conservative management in particular cases of asymptomatic and medically stable children with VGAMs.
\end{abstract}

https://thejns.org/doi/abs/10.3171/CASE20171

KEYWORDS vein of Galen malformation; congenital vascular conditions; pediatric disorders; spontaneous regression

Vein of Galen aneurysmal malformations (VGAMs) are congenital intracranial vascular lesions first described in 1937.1,2 VGAMs represent $30 \%$ of all pediatric vascular anomalies ${ }^{3}$ with an incidence of approximately 1 in 25,000 individuals. ${ }^{4-6}$ VGAMs present generally during the neonatal period or during infancy with congestive heart failure and intracranial hypertension. VGAM results from an arteriovenous connection between the median prosencephalic vein of Markowski and the primitive choroidal vessels. This vascular connection develops between weeks 6 and 11 of gestation. The malformation occupies the subarachnoid space of the velum interpositum and quadrigeminal cistern. ${ }^{7}$

There are several different classifications of VGAM, with the most common being the Raybaud and Lasjaunias system. ${ }^{8}$ In this system, there are 3 types of VGAMs: (1) choroidal, (2) mural, and (3) secondary to increased blood inflow due to an arteriovenous malformation. In addition, Yaşargil also developed a classification system based on the type of shunt present. ${ }^{9}$ In this system, VGAMs are classified by direct shunts (type I), network-like shunts (type II), a combination of direct shunts and arterial network (type III), and cerebral arteriovenous malformations (type IV). Choroidal VGAMs represent $56 \%-76 \%$ of cases. ${ }^{10-12}$

The severity of symptoms is related to the degree of intracranial shunt with variability by age. Neonates present with cardiac decompensation secondary to a high-flow systemic shunt. ${ }^{13-16}$ During infancy, increased head circumference secondary to venous hypertension and hydrocephalus is commonly observed. In these patients, there is a risk of spontaneous hemorrhage, seizure, and infarcts secondary to venous engorgement or blood flow diversion. Given the severity of the symptoms and the high mortality associated with untreated VGAMs (76.7\% mortality rate) and open surgical intervention ( $84.6 \%$ mortality rate), early endovascular treatment is recommended ( $15.7 \%$ mortality rate) ${ }^{17}$

VGAMs can also thrombose, a relatively rare event with a variable clinical outcome ranging from no symptoms to death. ${ }^{18}$ The proposed mechanism of thrombosis is the consequence of a low-flow shunt and

ABBREVIATIONS CT = computed tomography; MRI = magnetic resonance imaging; VGAM = vein of Galen aneurysmal malformation INCLUDE WHEN CITING Published February 22, 2021; DOI: 10.3171/CASE20171.

SUBMITTED December 22, 2020. ACCEPTED January 8, 2021.

(C) 2021 The authors, CC BY-NC-ND 4.0 (http://creativecommons.org/licenses/by-nc-nd/4.0/) 


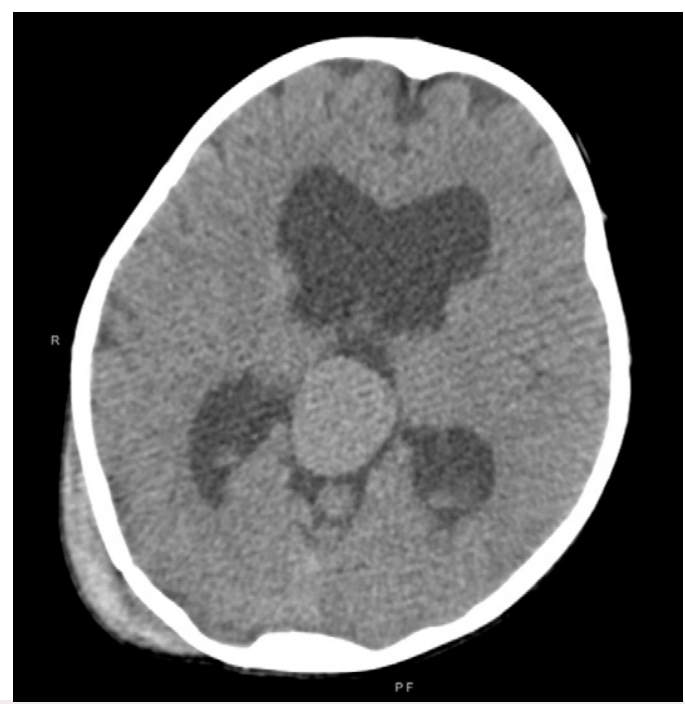

FIG. 1. CT of the head at presentation. Axial CT revealing moderate ventriculomegaly with a high-density mass at the pineal region suggestive of VGAM. A right parieto-occipital scalp hematoma and an underlying calvarial fracture are present.

insufficient venous drainage. In these cases, angiography of thrombosed VGAMs fails to identify the lesion, and there is an absence of flow. In addition, thrombosed VGAMs can appear hyperdense when visualized on computed tomography (CT) and exhibit centralized methemoglobin with a hemosiderin periphery on magnetic resonance imaging (MRI). ${ }^{19}$ If discovered after thrombosis, VGAMs may mimic pineal tumors, cavum vergae, pineal tumors, or porencephalic cysts on imaging. ${ }^{20,21}$ There have been limited reports on the successful surgical intervention of thrombosed VGAMs. ${ }^{22-25}$ However, cases of spontaneous disappearance and regression of VGAMs are extremely rare in the literature. ${ }^{26}$

\section{Illustrative Case}

We present the case of a 31-month-old female who presented with an incidentally found VGAM. The patient initially presented for evaluation after a head trauma secondary to a fall down a flight of stairs onto concrete. The patient was neurologically intact at presentation. CT of the head performed in the emergency department revealed a right parieto-occipital nondisplaced skull fracture extending to the lambdoid suture and moderate ventriculomegaly (Fig. 1). Subsequent MRI was notable for a large VGAM with associated hydrocephalus due to compression of the cerebral aqueduct by a feeding artery (Fig. 2). Formal cerebral angiography was then performed, identifying a vascular shunt with multiple feeders arising from the medial and lateral posterior choroidal arteries (Fig. 3). There was no arterial contribution from the anterior cerebral artery or anterior choroidal artery. Angiography also revealed a single-hole fistula used by all of the arterial feeders along the anterior aspect of the vein of Galen aneurysmal dilatation. The patient was formally evaluated by a cardiologist, who identified a patent foramen ovale without evidence of congestive heart failure. After initial evaluation, the patient was seen regularly in follow-up for 4 years, during which she had no evidence of increased head circumference, developmental limitations, or congestive heart failure. The patient was referred for consideration of elective embolization for her VGAM but was lost to follow-up for approximately 6 years. She returned to the clinic at age 12 years for a workup for a new frontal headache. MRI/magnetic resonance angiography performed at that time revealed the absence of the previously identified VGAM, thought to represent delayed involution of the lesion (Fig. 4). There was interval improvement of previously noted hydrocephalus and new homogeneous prominence of the pituitary gland thought to represent physiological pubertal pituitary hyperplasia. Ophthalmological evaluation of her symptoms disclosed a visual refractive error that was treated with prescription eyeglasses, relieving her headache.

\section{Discussion \\ Observations}

This case represents a rare occurrence of the spontaneous resolution of an identified VGAM. To our knowledge, there are very few cases of this event reported in the literature. ${ }^{27,28}$ Given the high degree of morbidity and mortality in children with VGAMs, clinicians seek to intervene early in the disease course. Management is guided by the criteria developed by Hôpital de Bicêtre in France. ${ }^{8}$ Under these guidelines, neonates with congestive heart failure are treated with aggressive medical therapy until evaluation for endovascular
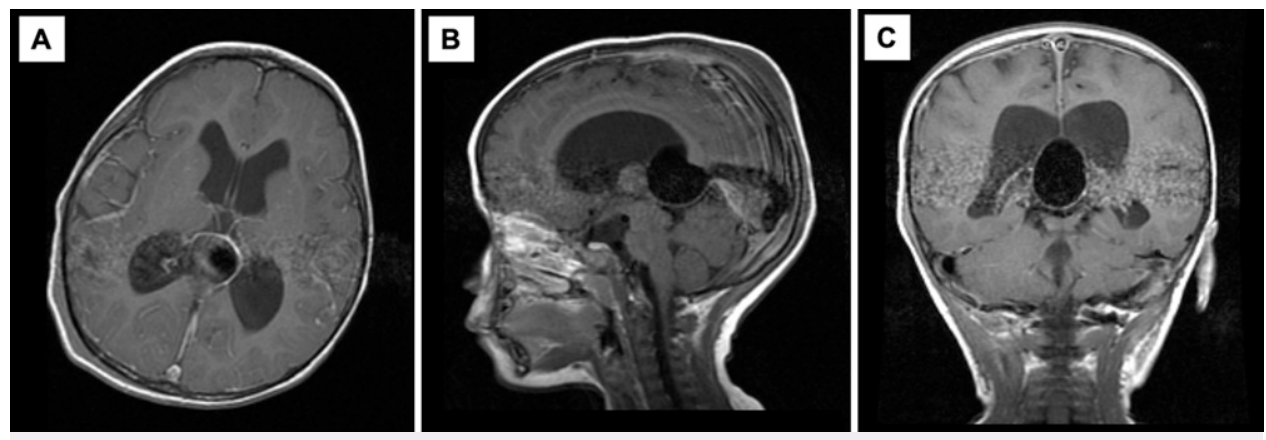

FIG. 2. MRI/magnetic resonance angiography (MRA) of the brain with contrast at presentation. A large VGAM measuring $3.2 \times 2.4 \mathrm{~cm}$ is present in the pineal region. The lesion was associated with prominent arterial feeders arising from the posterior circulation and an enlarged anomalous straight sinus. Previously noted moderate ventriculomegaly of the lateral and third ventricles visualized by CT was stable. A: Axial view. B: Sagittal view. C: Coronal view. 

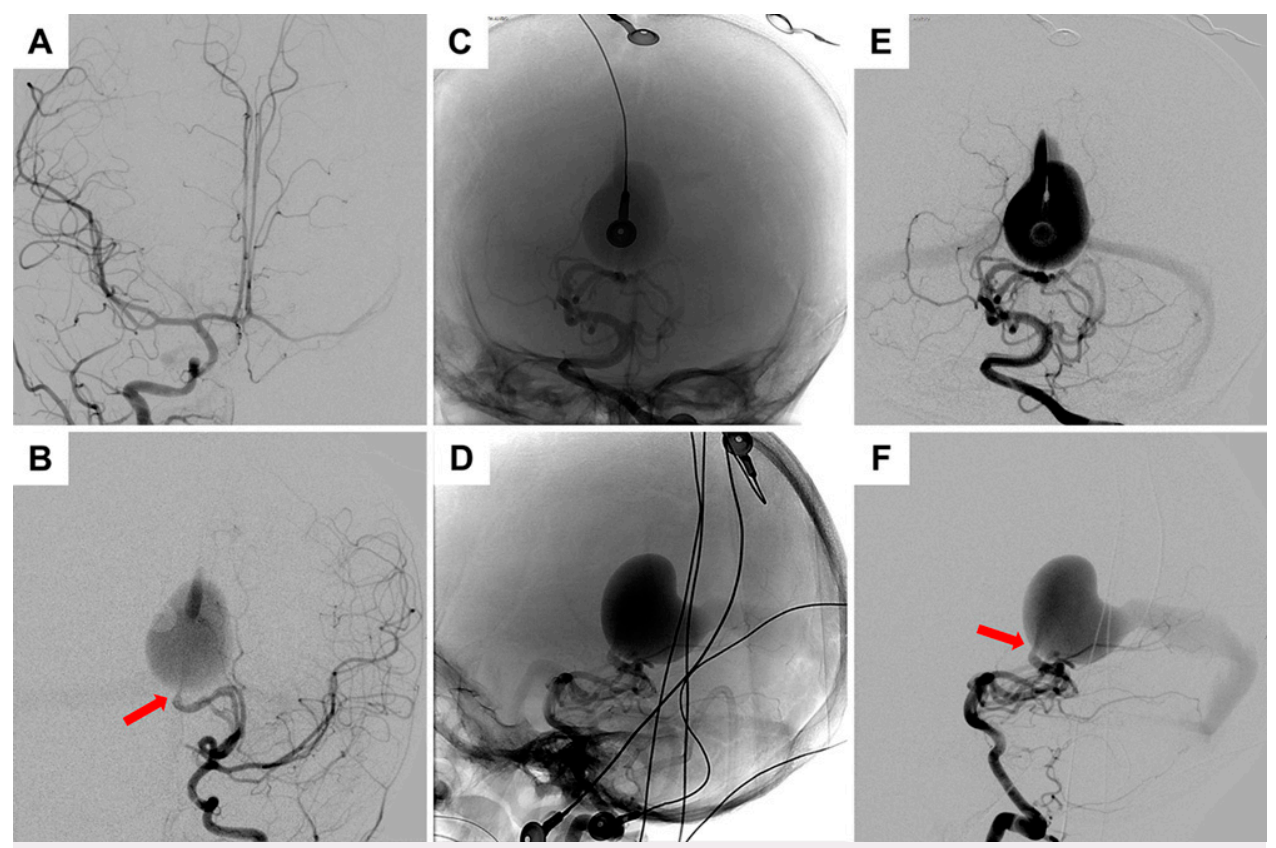

FIG. 3. Digital subtraction angiography of the patient. VGAM was identified with multiple feeders arising from the medial and lateral posterior choroidal arteries. A single-hole fistula was noted along the anterior aspect of the VGAM (red arrows). Venous drainage of the vein of Galen occurs via a median vein of the prosencephalon, falcine sinus. A: Right internal carotid artery injection (anteroposterior [AP] view). B: Left internal carotid artery injection (AP view). C-F: Left vertebral artery injections with AP and lateral projections, with both unsubtracted and subtracted images demonstrating artifact from monitoring leads.
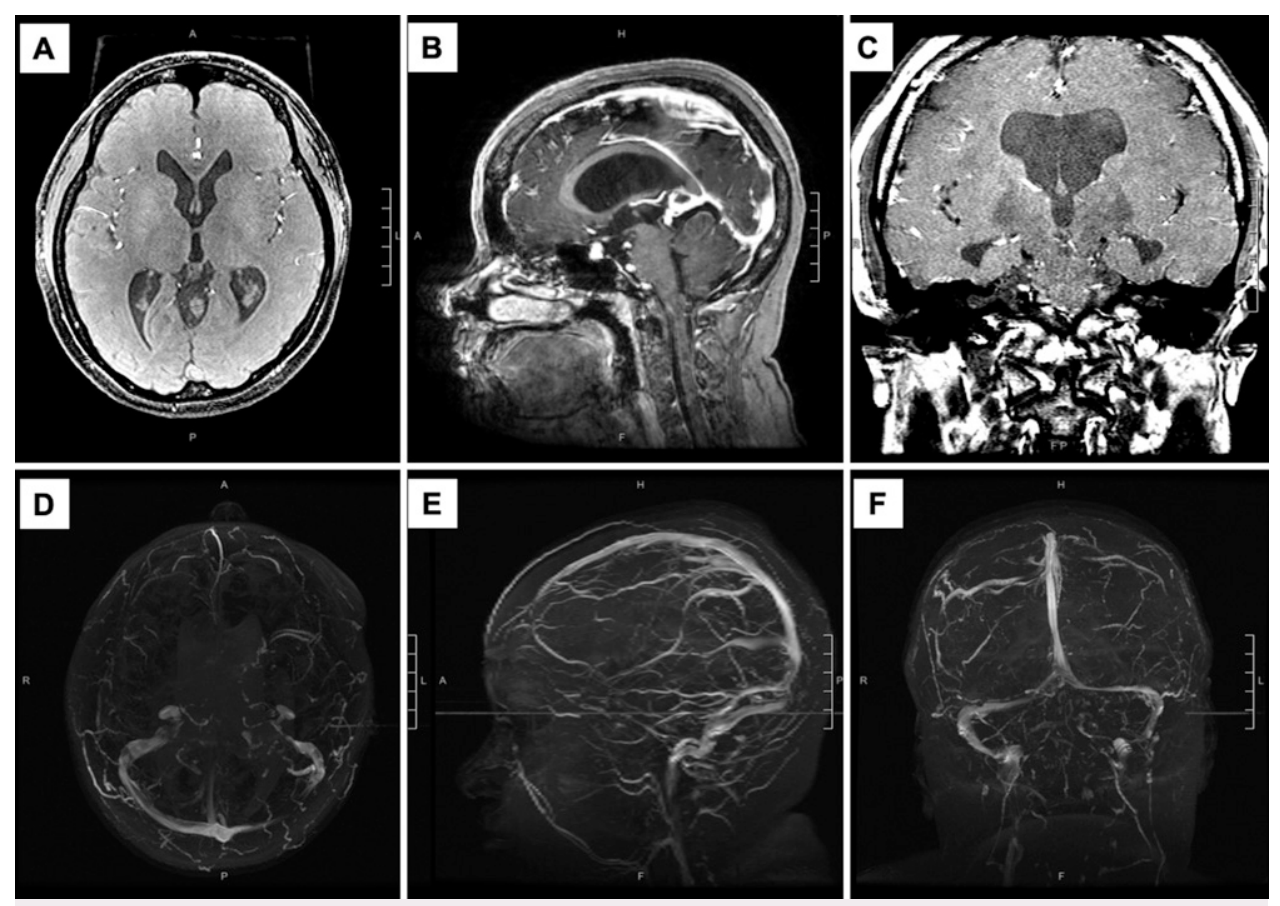

FIG. 4. MRI/MRA of the brain with contrast at the long-term follow-up. Previously seen VGAM/ promesencephalic venous complex is no longer present. Mild ventriculomegaly was improved from prior imaging. The straight sinus is now visible. A: Axial MRI. B: Sagittal MRI. C: Coronal MRI. D: Axial MRA. E: Sagittal MRA. F: Coronal MRA. 
intervention can be completed. In cases with severe multiorgan failure or brain damage, therapy may be withheld. The patient described in the present report is one of a handful of unique cases that demonstrated complete regression of the lesion in a largely asymptomatic patient. Importantly, previous studies of VGAMs noted that the presence of major medical comorbidities was associated with a poor prognosis, none of which were present in this patient. At presentation, the patient had a maximum Bicêtre score of 21, which recommends delayed embolization in this clinical scenario. ${ }^{29,30}$ The primary goal of endovascular intervention is to preserve life and prevent developmental delay. Fortunately, the discussed patient met her appropriate developmental milestones and was progressing through school without issue. Although her VGAM completely regressed, the persistence of some mild hydrocephalus likely reflects a combination of impaired cerebrospinal fluid resorption and a degree of volume loss in white matter. ${ }^{30}$ This finding is consistent with previous observations that, although hydrocephalus can result from obstruction by vein of Galen aneurysmal dilatation, a major component is secondary to a hydrodynamic process that increases venous pressure. ${ }^{31}$ Although hydrocephalus should be monitored as the patient continues to mature, there is evidence that the treatment of hydrocephalus in cases of VGAM is associated with a high risk of complications, even after embolization. ${ }^{32}$

\section{Lessons}

This case adds to the few reported cases demonstrating spontaneous resolution of VGAMs in the literature. Current clinical guidelines seek early endovascular intervention in neonates and infants in order to minimize mortality and maximize neurodevelopmental outcome. However, this particular patient had her VGAM incidentally discovered during a trauma evaluation and was otherwise asymptomatic from the lesion. Although the intent was to electively treat this patient with endovascular intervention, the loss of the patient to follow-up and subsequent spontaneous resolution of the lesion is a notable finding. Thus, it remains possible that there exists a subset of VGAMs that are asymptomatic and undetected and that subsequently thrombose and regress within the pediatric population. Future studies may benefit from exploring imaging and angiographic findings that could predict which VGAMs may resolve in a similar manner. These studies may inform future management of asymptomatic patients with VGAM without evidence of associated medical comorbidities.

\section{Acknowledgments}

This work was supported by the National Institute of Neurological Disorders and Stroke Research Education Grant (NIH 5R25NS065741-09 to K.K.K.).

\section{References}

1. Mahmoodi R, Habibi Z, Heidari V, et al. Spontaneous regression and complete disappearance of the vein of Galen aneurysmal malformation. Childs Nerv Syst. 2016;32(4):593-598

2. Jaeger JR, Forbes RP, Dandy WE. Bilateral congenital cerebral arteriovenous communication aneurysm. Trans Am Neurol Assoc. 1937;63:173-176.

3. Long DM, Seljeskog EL, Chou SN, et al. Giant arteriovenous malformations of infancy and childhood. J Neurosurg. 1974;40(3): 304-312.

4. Li AH, Armstrong D, terBrugge KG. Endovascular treatment of vein of Galen aneurysmal malformation: management strategy and 21-year experience in Toronto. J Neurosurg Pediatr. 2011;7(1):3-10.
5. Bhattacharya JJ, Thammaroj J. Vein of Galen malformations. J Neurol Neurosurg Psychiatry. 2003;74(suppl 1):i42-i44.

6. Frawley GP, Dargaville PA, Mitchell PJ, et al. Clinical course and medical management of neonates with severe cardiac failure related to vein of Galen malformation. Arch Dis Child Fetal Neonatal Ed. 2002;87(2):F144-F149.

7. Gupta AK, Rao VR, Varma DR, et al. Evaluation, management, and long-term follow up of vein of Galen malformations. J Neurosurg. 2006;105(1):26-33.

8. Lasjaunias P. Vein of Galen aneurysmal malformation. In: Lasjaunias P, ed. Vascular Diseases in Neonates, Infants and Children: Interventional Neuroradiology Management. Springer; 1997:67-202.

9. Gailloud P, O'Riordan DP, Burger I, et al. Diagnosis and management of vein of Galen aneurysmal malformations. J Perinatol. 2005;25(8):542-551.

10. Fullerton HJ, Aminoff AR, Ferriero DM, et al. Neurodevelopmental outcome after endovascular treatment of vein of Galen malformations. Neurology. 2003;61(10):1386-1390

11. Lasjaunias P, Rodesch G, Terbrugge K, et al. Vein of Galen aneurysmal malformations. Report of 36 cases managed between 1982 and 1988. Acta Neurochir (Wien). 1989;99(1-2):26-37.

12. Meyers PM, Halbach VV, Phatouros CP, et al. Hemorrhagic complications in vein of Galen malformations. Ann Neurol. 2000; 47(6):748-755.

13. Amacher AL, Shillito J Jr. The syndromes and surgical treatment of aneurysms of the great vein of Galen. J Neurosurg. 1973;39(1): 89-98.

14. Gold A, Ransohoff J, Carter S. Vein of Galen malformation. Acta Neurol Scand Suppl. 1964;40(suppl 11):1-31.

15. Hirano A, Solomon S. Arteriovenous aneurysm of the vein of Galen. Arch Neurol. 1960;3:589-593.

16. Maheut J, Santini JJ, Barthez MA, et al. Clinical symptomatology in aneurysm of the ampulla of Galen. Results of a national survey. Article in French. Neurochirurgie. 1987; 33(4):285-290.

17. Khullar D, Andeejani AM, Bulsara KR. Evolution of treatment options for vein of Galen malformations. J Neurosurg Pediatr. 2010; 6(5):444-451.

18. Moftakhar P, Danielpour M, Maya M, et al. Spontaneous thrombosis of neonatal vein of Galen malformation. Neurosurg Focus. 2009;27(5):E12.

19. Abrão GP, Barbosa LA, Sá AT Jr, etal. Spontaneous thrombosis of a malformation of the vein of Galen. Arq Neuropsiquiatr. 2010;68(4): 648-650.

20. Ahmad M, Mir S, Wahab S, et al. Vein of Galen malformation. BMJ Case Rep. 2012;2012:bcr2012006822.

21. Gokhale S, Laskowitz DT. Teaching neuroimages: vein of Galen aneurysm mimicking pineal mass in a young adult. Neurology. 2013;80(22):e240

22. Weir BK, Allen PB, Miller JD. Excision of thrombosed vein of Galen aneurysm in an infant. Case report. J Neurosurg. 1968;29(6): 619-622.

23. Lazar ML. Vein of Galen aneurysm: successful excision of a completely thrombosed aneurysm in an infant. Surg Neurol. 1974 2(1):22-24.

24. Di Rocco C, lannelli A, Puca A, et al. Spontaneous thrombosis of an aneurysm of the great vein of Galen. Eur Neurol. 1983;22(4): 293-299.

25. Konovalov AN, Pitskhelauri DI, Arutiounov NV. Surgical treatment of the thrombosed vein of Galen aneurysm. Acta Neurochir (Wien) 2002;144(9):909-915.

26. Lasjaunias PL, Chng SM, Sachet M, et al. The management of vein of Galen aneurysmal malformations. Neurosurgery. 2006; 59(suppl 3):S184-S194. 
27. Lasjaunias $P$, Hui $F$, Zerah $M$, et al. Cerebral arteriovenous malformations in children. Management of 179 consecutive cases and review of the literature. Childs Nerv Syst. 1995;11(2):66-79.

28. Konus OL, Ilgit ET, Ozdemir A, et al. Spontaneous thrombosis of a vein of Galen aneurysmal malformation: possible effects of contrast media. Eur Radiol. 2000;10(9):1456-1458.

29. Hoffman HJ, Chuang S, Hendrick EB, et al. Aneurysms of the vein of Galen. Experience at The Hospital for Sick Children, Toronto. J Neurosurg. 1982;57(3):316-322.

30. McSweeney N, Brew S, Bhate S, et al. Management and outcome of vein of Galen malformation. Arch Dis Child. 2010;95(11): 903-909.

31. Zerah M, Garcia-Monaco R, Rodesch G, et al. Hydrodynamics in vein of Galen malformations. Childs Nerv Syst. 1992;8(3):111-117.

32. Schneider SJ, Wisoff JS, Epstein FJ. Complications of ventriculoperitoneal shunt procedures or hydrocephalus associated with vein of Galen malformations in childhood. Neurosurgery. 1992;30(5):706-708.

\section{Disclosures}

The authors report no conflict of interest concerning the materials or methods used in this study or the findings specified in this paper.

\section{Author Contributions}

Conception and design: Hong, Kumar. Acquisition of data: all authors. Analysis and interpretation of data: all authors. Drafting the article: Kumar, Dodd. Critically revising the article: all authors. Reviewed submitted version of manuscript: all authors. Approved the final version of the manuscript on behalf of all authors: Hong. Study supervision: Hong.

\section{Correspondence}

David S. Hong: Stanford University, Stanford, CA. dsh1@ stanford.edu. 